\title{
An experimental study of wall-injected flows in a rectangular cylinder
}

\author{
A. Perrotta - G.P. Romano - B. Favini
}

Received: date / Accepted: date

\begin{abstract}
An experimental investigation of the flow inside a rectangular cylinder with air injected continuously along the wall is performed. This kind of flow is a two-dimensional approximation of what happens inside a solid rocket motor, where the lateral grain burns expelling exhaust gas or in processes with air filtration or devices to attain uniform flows. We propose a brief derivation of some analytical solutions and a comparison between these solutions and experimental data, which are obtained using the Particle Image Velocimetry (PIV) technique, in order to provide a global reconstruction of the flowfield. The flow, which enters orthogonal to the injecting wall, turns suddenly its direction being pushed towards the exit of the chamber. Under the incompressible and inviscid flow hypothesis, two analytical solutions are reported and compared. The first one, known as Hart-McClure solution, is irrotational and the injection velocity is non-perpendicular to the injecting wall. The other one, due to Taylor and Culick, has non-zero vorticity and constant, vertical injection velocity. The comparison with laminar solutions is useful to assess whether transition to turbulence is reached and how the disturbance thrown in by the porous injection influences and modifies those solutions.
\end{abstract}

Keywords Wall-injected flows · PIV · Confined flows

PACS 47.10.A- * 47.27.Cn $\cdot 47.27 . n d \cdot 47.56 .+\mathrm{r}$

\section{List of symbols}

A

$A_{0}$

$B_{j}$

$H$

I

A. Perrotta (৫) · G.P. Romano · B. Favini

Department of Mechanical and Aerospace Engineering

Sapienza - University of Rome, Rome, Italy

E-mail: andrea.perrotta@uniroma1.it area of the porous plate; $A=120 \mathrm{~cm}^{2}$ amplitude of acoustic waves $j$-th bin of the histogram representation height of the channel; $H=2 \mathrm{~cm}$ turbulence intensity; $I=\sqrt{\left\langle u^{\prime} u^{\prime}\right\rangle+\left\langle v^{\prime} v^{\prime}\right\rangle}$ 
turbulence intensity peak; $\max _{y} I$ length of the channel; $L=24 \mathrm{~cm}$ total number of bins
of $\nabla \mathbf{u} ; P=\frac{\partial u}{\partial x}+\frac{\partial v}{\partial y}$ $L$ $N_{\text {bin }}$ $P \quad$ divergence of a two-dimensional flow, first invariant of $\nabla \mathbf{u} ; P=\frac{\partial u}{\partial x}+\frac{\partial v}{\partial y}$ $Q \quad$ third invariant of $\nabla \mathbf{u} ; Q=\frac{\partial u}{\partial x} \frac{\partial v}{\partial y}-\frac{\partial u}{\partial y} \frac{\partial v}{\partial x}$ $U_{\text {inj }}$ injection velocity

$\Delta$ discriminant of the eigenvalues characteristic equation for the two-dimensional $\nabla \mathbf{u} ; \Delta=P^{2}-4 Q$

$\Omega_{z}$

transversal component of vorticity - $\quad$ spatial average; i.e. a discrete approximation of the integral $\frac{1}{L} \int_{0}^{L} s(x) d x$

$\therefore$

.$*$

$\dot{m}$

$\lambda$

$\langle\cdot\rangle$ $\mathrm{Re}_{\mathrm{inj}}$ $\nu$

$\psi$

$\Omega$

$\mathbf{u}$

$h_{i}$

$k$

$p$

$u$

$v$

\section{Introduction}

This paper presents an experimental study of the the flow inside a rectangular cylinder with one of the two long sides injecting air through a porous plate. This kind of flow represents a two-dimensional approximation of what happens in a solid or hybrid rocket motor or in the flow of filtered gas in heat exchangers or micromixers. The first study of a wall-injected flow inside a rectangular cylinder is due to Taylor (1956), who studied injection from the inner wall of a cone and a wedge that admit respectively as limit cases a cylinder and a parallelepiped. Also Culick (1966) and Varapaev and Yagodkin (1969) proposed a steady solution, i.e. the mean flow, of the incompressible and inviscid model, respectively for the cylindrical and planar configuration. These solutions were found under the same hypotesis of non-zero vorticity, and we will refer to both as Taylor-Culick (TC). Earlier, an irrotational solution was proposed by Hart and McClure (1959) and McClure 
et al (1963), referred to as Hart-McClure (HC). Both TC and HC models can be numerically integrated when viscosity is taken into account. More complex solutions exist for the cylindrical configuration, see Majdalani and Fist (2014), but they do not have a planar counterpart and therefore they are not discussed here.

For high injection Reynolds number, $\operatorname{Re}_{\mathrm{inj}}=H U_{\mathrm{inj}} / \nu$, with $H$ the height of the channel, $U_{\text {inj }}$ the injection velocity and $\nu$ the kinematic viscosity, between 675 and 2500, theoretical studies of linear stability were carried out on the TC mean flow by Casalis et al (1998) and Griffond et al (2000), by superposing a normal perturbation. Even though a normal perturbation is not consistent with a nonparallel flow, it fits very well with experimental results obtained by Avalon et al (1998) and Griffond et al (2000) with the VECLA facility, at the ONERA-Palaiseau laboratory, by means of hot wire measurements. Also PLIF visualizations of the hydrodynamic instabilities have been conducted by Avalon et al (2001) with the VECLA: a rectangular cylinder with optical access on one side and air injected from the bottom through a porous plate. A feature of this linear stability analysis is that, for a given unstable mode and position, there is a continuous range of unstable frequencies. A discrete spectrum is, instead, yielded by a technique that does not make any assumption on the longitudinal shape of the eigenfunctions as stated by Féraille and Casalis (2005), Casalis et al (2006), Chedevergne et al (2006). Anyhow, only temporally damped modes are obtained, but the shape of the eigenmodes, which show a quasi-exponential growth in space, is thought to be the cause of hydrodynamic instability. Indeed, according to Culick (1966) and Flandro (1986), hydrodynamic instability is activated when amplitude of perturbation and acoustic frequencies of the chamber match, in some sense.

From the theoretical study of Beddini (1986), based on a broad range of Reynolds numbers, emerges that the relation between injection Reynolds number and position at which instability or transition occurs, depends on the pseudoturbulence parameter $\sqrt{\left\langle v^{\prime} v^{\prime}\right\rangle}$, that is the rms of the injection velocity. This is related to the non-uniformity of the injected velocity from the porous medium, as shown by Pimenta and Moffat (1974) and Perrotta et al (2017). Indeed, the study performed by Yagodkin (1980) does not predict transition for injection Reynolds numbers ranging from 4 to 125, which was, instead, observed by Olson and Eckert (1966) and Huesmann and Eckert (1968). Beddini describes three regimes when moving downstream into the cylinder: laminar, transitional and fully turbulent. In the first two regimes, the mean velocity profiles correspond to the one provided by the laminar theory, but the transitional one is characterized by the presence of pronounced turbulence intensity peaks. The third regime is furthermore characterized by a change in the shape of the mean velocity profiles.

Herein, we investigate the wall-injected flow, with an injection Reynolds number around 100, by means of Particle Image Velocimetry (PIV), in order to assess the relation between disturbances introduced by the injection and transition of the mean flow from the first to the second regime described above. PIV is particularly indicated because it is non-intrusive and provides a global and accurate view of the velocity field in the whole observed region, see also Laboureur et al (2010). The mean flow is reconstructed and the statistics of the fluctuations around the mean field is derived, in order to investigate the basic mechanisms leading to departures from the laminar theoretical approximation presented in the next section. This is the main objective of this work, together with the detailed investigation 
on the inlet flow behaviour downstream the porous plate. At the present Reynolds numbers, it is possible to attain very high spatial resolution with Particle Image Velocimetry, thus allowing a detailed description of the flow features after the grain section of the porous plate.

The paper is structured as follows. Sect. 2 shows the analytical reference solutions. Then, Sect. 3 describes the experimental set-up and in Sect. 4 experimental data are analyzed. Finally, conclusions and future works are provided in Sect. 5.

\section{Analytical solutions}

In order to find an analytical solution of the mean flow, as already stated in the introduction, we consider the incompressible and inviscid Navier-Stokes equations

$$
\begin{aligned}
& \nabla \cdot \mathbf{u}=0 \\
& (\mathbf{u} \cdot \nabla) \mathbf{u}=-\nabla p .
\end{aligned}
$$

The simplest solution, which is due to Hart-McClure, makes the irrotational assumption $\nabla \times \mathbf{u}=0$. Let us assume a planar, two-dimensional configuration as shown in Fig. 1 so that the stream function, $\psi$, is proved to exist. Here, superscripted variables have dimensions while dimensionless variables are defined as follows

$$
(x, y, z)=\frac{\left(x^{*}, y^{*}, z^{*}\right)}{H} \quad \text { and } \quad \mathbf{u}=\frac{\mathbf{u}^{*}}{U_{\mathrm{inj}}},
$$

where $U_{\text {inj }}$ is the injection velocity from the wall and $H$ is the height of the rectangular cylinder as sketched in Fig 1. In this section, each variable is dimensionless, but in the following we will omit superscripts and it will be clear from the context whether variables have dimensions or not. From the stream function, the two components of the velocity field are obtained as $u=\partial \psi / \partial y$ and $v=-\partial \psi / \partial z$. The conservation of mass implies

$$
\int_{-1}^{0} \frac{\partial \psi}{\partial y} d y=x
$$

then $\partial \psi / \partial y$ should be a linear function of $x$ and can be written as

$$
\frac{\partial \psi}{\partial y}=x \alpha(y)+\beta(y) \Longrightarrow \psi(x, y)=x \int \alpha(y) d y+\int \beta(y) d y+\gamma(x)
$$
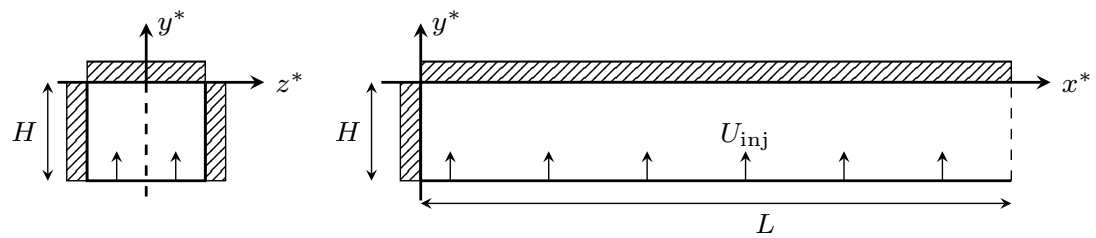

Fig. 1: Sketch of the planar configuration and reference frame 
with $\alpha(y)$ and $\beta(y)$ two unknown functions such that

$$
\begin{aligned}
& \int_{-1}^{0} \alpha(y) d y=1 \\
& \int_{-1}^{0} \beta(y) d y=0
\end{aligned}
$$

The zero vorticity hypothesis can be expressed in terms of the stream function

$$
\Omega_{z}=\nabla^{2} \psi=0
$$

that, thanks to (5), yields

$$
0 \equiv \frac{\partial^{2} \psi}{\partial x^{2}}+\frac{\partial^{2} \psi}{\partial y^{2}}=\gamma^{\prime \prime}(x)+x \alpha^{\prime}(y)+\beta^{\prime}(y) \Longrightarrow \beta^{\prime}(y)=-\gamma^{\prime \prime}(0) \quad \text { for all } y .
$$

Since

$$
\gamma^{\prime \prime}(x)=-\frac{\partial v}{\partial x}(x,-1)=-\frac{\partial U_{\mathrm{inj}}}{\partial x} \equiv 0,
$$

$\beta^{\prime}(y)$ equals zero for all $y \in[-1,0]$. Furthermore, thanks to Eq. (7), $\beta$ has zero integral, hence $\beta(y) \equiv 0$. Up to an additive constant, see Eq. (5) and (9), we can choose the stream function as $\psi(x, y)=x F(y)^{1}$. Thus, Equation (8) becomes an ordinary differential equation in $F$

$$
x F^{\prime \prime}(y)=0 \Longrightarrow F(y)=A y+B .
$$

Now, imposing the boundary conditions

$$
\left\{\begin{array}{l}
v(y=0)=0 \\
v(y= \pm 1)=\mp 1
\end{array}\right.
$$

yields $B=0$ and $A=1$. Then

$$
u=x \quad \text { and } \quad v=-y \text {. }
$$

These are the predicted non-dimensional, velocity components under the hypothesis of irrotational flow. Note that this solution has oblique injection velocity at the wall. This will be referred to as HC solution.

On the other hand, Varapaev and Yagodkin, under the previous hypothesis on the stream function, did not impose the vorticity to be zero, but solved the vorticity equation $\nabla \times(\mathbf{u} \times \boldsymbol{\Omega})=0$, which is actually scalar, with constant injection velocity and impermeability at the upper wall,

$$
\left\{\begin{aligned}
x\left(F F^{\prime \prime \prime}-F^{\prime} F^{\prime \prime}\right) & =0 \\
v(x,-1)=-F(-1) & =1 \\
v(x, 0)=-F(0) & =0 \\
u(x,-1)=F^{\prime}(-1) & =0,
\end{aligned}\right.
$$

\footnotetext{
1 We proved that this form of $\psi$ is a consequence of the conservation of mass under the zero vorticity hypothesis. Without this hypothesis we are not able to prove that $\beta$ is zero.
} 
which admits the non-unique solution

$$
F(y)=(-1)^{n} \sin \left[\left(\frac{\pi}{2}+n \pi\right) y\right] .
$$

The only physical solution, which gives a radial velocity vanishing only on the axis, is the case with $n=0$, the other ones being excluded as unphysical. Therefore

$$
u=\frac{\pi x}{2} \cos \left(\frac{\pi y}{2}\right) \quad \text { and } \quad v=-\sin \left(\frac{\pi y}{2}\right) .
$$

This is the solution of non-dimensional, velocity components for the rotational flow condition to be referred as TC solution. Note that $\nabla \times \mathbf{u} \neq 0$, in fact

$$
\Omega_{z}=\nabla^{2} \psi=-\frac{x \pi^{2}}{4} \sin \left(\frac{\pi y}{2}\right) .
$$

A third approximation is to consider rotational, viscous conditions so that Eq. (13) becomes

$$
\left\{\begin{aligned}
F F^{\prime \prime \prime}-F^{\prime} F^{\prime \prime} & =\operatorname{Re}_{\mathrm{inj}}^{-1} F^{\prime \prime \prime \prime} \\
v(x,-1)=-F(-1) & =1 \\
v(x, 0)=-F(0) & =0 \\
u(x,-1)=F^{\prime}(-1) & =0 \\
u(x, 0)=F^{\prime}(0) & =0
\end{aligned}\right.
$$

when the no-slip condition is imposed at the wall in $y=0$. While Eqs. (12) and (15) are analytical solutions, Equation (17) can be solved numerically.

\section{Experimental set-up}

In order to reproduce the flow configuration described in Fig. 1, a rectangular cylinder has been considered. It is mostly made of Plexiglas to allow optical access and has an injecting-wall made of a porous Polyethylene plate, with a porosityi.e. the maximum size of the pores - of $20 \mu \mathrm{m}$. Another feature of porous materials is the void fraction or open-area ratio, i.e. hole-area/total-area, that is not directly available as in homogenous screens. Anyhow, the structure of a Polyethylene plate exhibits several homogenous spheres sintered together, therefore the void fraction should correspond to the empty space between these spheres. Let $d_{\mathrm{s}}$ be the diameter of one sphere and $L \times H$ the sample dimensions, then a two-dimensional array of spheres occupies a percentage of the total area equal to $\frac{H-d_{\mathrm{s}}}{H} \frac{\pi}{2 \sqrt{3}} \rightarrow 0.9$ as $H$ tends to infinity, which is furthermore independent on the diameter of the sphere. Therefore, the void fraction is about $10 \%$ and does not depend on the porosity. Note that this approximate model of flanked spheres, overestimates the nominal porosity, in fact the biggest sphere able to pass through the empty space between three spheres has radius equal to $\left(\sqrt{3} / 2-\frac{1}{2} \tan (\pi / 6)-1 / 2\right) d_{\mathrm{s}}$. Taking $d_{\mathrm{s}}=0.2 \mathrm{~mm}$, i.e. the average diameter of spheres into a $20 \mu \mathrm{m}$-porosity plate, this yields a porosity of around $30 \mu \mathrm{m}$.

The flow is injected by four inlets into a pre-chamber to allow uniform spatial distribution before passing through the porous plate. A compressor feeds this prechamber with premixed air and oil droplets working as seeding particles for PIV. 
The diameters of such particles varying between 1 to $5 \mu \mathrm{m}$, as generated by a Laskin nozzle. The flow rate injected into the chamber is imposed by the exit pressure from the compressor, equal to 1 bar, which corresponds to a volume flow rate of $\dot{m}=1.117 \times 10^{-3} \mathrm{~m}^{3} \mathrm{~s}^{-1}$ at the feeding tube. Thanks to conservation of mass, an injection velocity $U_{\mathrm{inj}}=\dot{m} / A \approx 9.30 \mathrm{~cm} \mathrm{~s}^{-1}$ is achieved, where $A=120 \mathrm{~cm}^{2}$ is the area of the the porous plate, and hence an injection Reynolds number

$$
\operatorname{Re}_{\mathrm{inj}}=\frac{H U_{\mathrm{inj}}}{\nu}=\frac{0.02 \times 0.093}{1.568 \times 10^{-5}} \approx 114
$$

A cross-correlation camera acquires a couple of images each $0.2 \mathrm{~s}$, which is the acquisition rate of the whole system. Each frame is illuminated by a 1 to $2 \mathrm{~mm}$ wide laser sheet, which is generated by a Nd:Yag Laser and synchronized with the camera by a pulse generator. The laser sheet goes into the chamber from the closed side (which corresponds to $z=0 \mathrm{~cm}$ in Fig. 1) and such a plane is orthogonal to the camera, which records a region inside the chamber of $8.2 \mathrm{~cm}$ by $2 \mathrm{~cm}$ (i.e. the height of the chamber). Each image has a resolution of around $300 \mathrm{dpi}$ (118 pixel per $\mathrm{cm}$ ). A detailed scheme of the experimental set-up is shown in Fig. 2, where the location of the adopted devices is shown. Non-perfect alignment between camera and laser sheet would lead to blurred images, non-perfect alignment between optical system and injection direction would cause loss of tracers, yielding spurious vectors during the analysis. Therefore, orthogonality is a fundamental issue and has been ensured by the optical bench on which all the devices are fastened. As the width of the laser sheet is $1-2 \mathrm{~mm}$ and the dimensions of an image are $2-8 \mathrm{~cm}$, the maximum angle that a velocity vector can have with respect to the $(x, y)$-plane is $2.8^{\circ}$ leading to an error of $1-\cos \left(2.8^{\circ}\right) \approx 10^{-3}$ that is largely below the intrinsic error due to PIV algorithm.

In order to cover the whole length of the chamber, which equals $24 \mathrm{~cm}$, three partially overlapped sections are acquired, as indicated in Fig. 2 by the three numbered rectangles. For PIV processing, we used the Open-source software PIVlab devoloped by Thielicke and Stamhuis (2014), being these specific results almost coincident with those obtained with a commercial software. For each section, a sequence of 1000 couples is analyzed with decreasing window size, i.e. from $128 \times 128$ pixels to $16 \times 16$ pixels with a $50 \%$ overlapping, thus giving a velocity vector resolution of one vector each 8 pixels, corresponding to one vector each $0.5 \mathrm{~mm}$. A representation of the data grid is given in Fig. 3.

A preliminary analysis is carried out in order to characterize the injection profile of velocity near the porous plate also in view of a proper numerical simulation of inlet conditions. In order to minimize the effects on the velocity distribution at the outlet of the porous plate, during this preliminary test, the upper wall was removed and the exit on the right, at $x=24 \mathrm{~cm}$, was closed. For such a preliminary test, the time interval between frames, $\Delta t$, is equal to $6000 \mu \mathrm{s}$. For the test with the closed upper section, instead, $\Delta t$ has been changed accordingly to the expected linear increment of the axial velocity with $x$ (Eq. (12)) and its values are summarized in Table 1. A typical estimation of the error for PIV measurements is given by displacements of a tenth of a pixel per frame. Since values of this error in meter per second depend on $\Delta t$, they are reported in Tab. 1 too. 


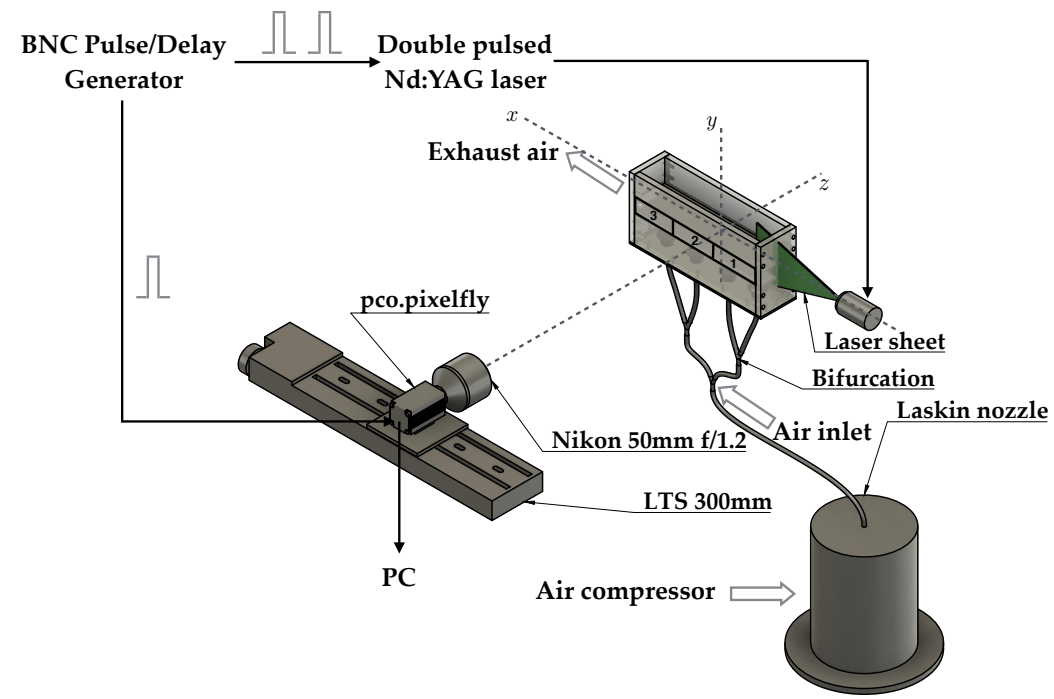

Fig. 2: Scheme of the experimental set-up

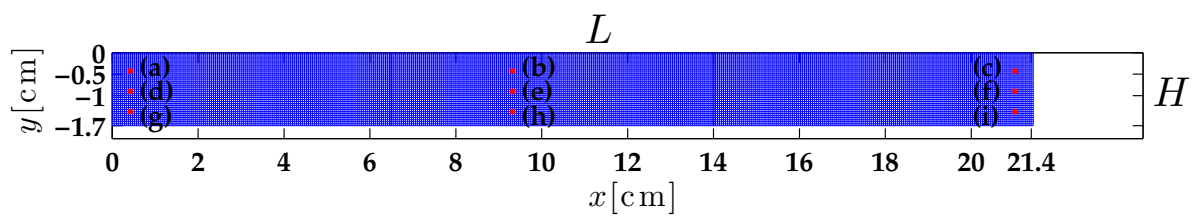

Fig. 3: Mesh obtained with the PIV software with highlighted nine points at which PDFs are calculated (Fig. 13 and 14)

\begin{tabular}{lll}
\hline Section & $\Delta t[\mu \mathrm{s}]$ & Error $[\mathrm{mm} / \mathrm{s}]$ \\
\hline 1 & 3000 & 1.9 \\
2 & 2000 & 2.9 \\
3 & 1000 & 5.8 \\
\hline
\end{tabular}

Table 1: Values of $\Delta t$ and error on velocity for each section in the closed-open configuration

\section{Results}

4.1 Inlet conditions on the plate

The porous plate is made of Polyethylene and has a porosity of $20 \mu \mathrm{m}$. Since tracer particles have diameters smaller than $5 \mu \mathrm{m}$, they easily pass through the filter as 


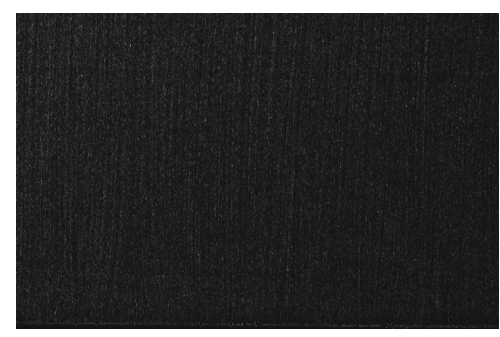

(a) $20 \mu \mathrm{m}$

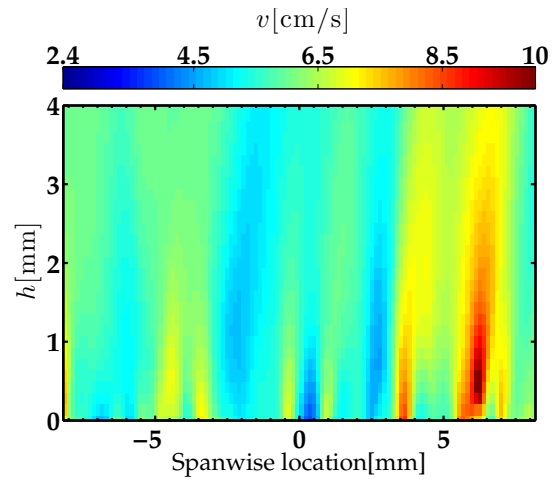

(c) $20 \mu \mathrm{m}$

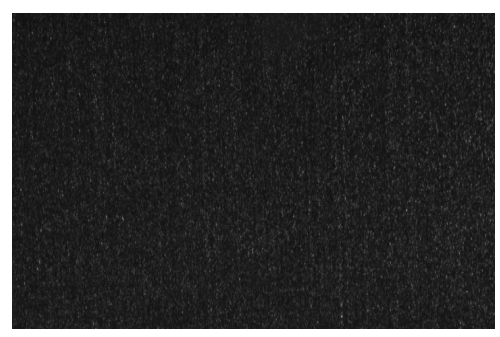

(b) $10 \mu \mathrm{m}$

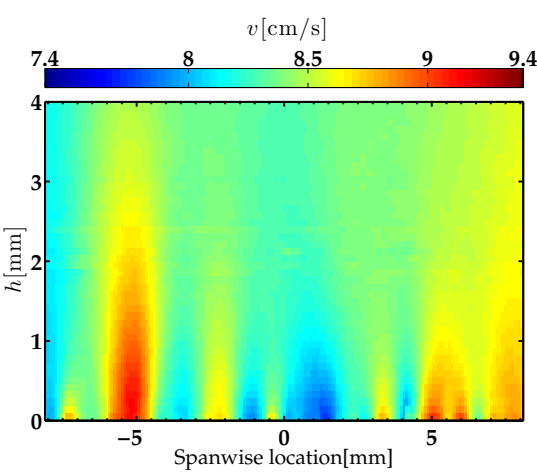

(d) $10 \mu \mathrm{m}$

Fig. 4: Raw images used for the PIV analysis (top) and their corresponding vertical velocity fields (bottom) obtained with two different porosities: $20 \mu \mathrm{m}$ (left) and $10 \mu \mathrm{m}$ (right)

clearly shown in Fig. 4, where two raw images (top) of the injected flow through two samples of different porosity, $20 \mu \mathrm{m}$ and $10 \mu \mathrm{m}$, are reported together with their corresponding vertical velocity fields (bottom). The preliminary characterization of the inlet flow indicates the presence of jet-like structures near the plate and of a mostly laminar flow far from it, as reported in Fig. 5. Since the filter has a junction at $x=12 \mathrm{~cm}$, around that abscissa, we note a region where the vertical velocity component is very small and the horizontal one is much higher because of such an obstacle. In the whole field, excluding the borders where the flow is much more influenced by external disturbances, the horizontal velocity component is small. However, jet-like structures are clearly visible in both velocity components, in the form of alternating high-speed and low-speed streaks down to a scale which is smaller than $1 \mathrm{~cm}$. In Fig. 6 the parallel jet configuration is highlighted by plotting the average cross product among the two velocity fluctuations, i.e. the tangential Reynolds stress. Clearly, the effects of such jets propagates downstream of the porous plate. We can estimate, with this analysis, the spatial organization of the perturbation yielded by this porous plate. In Fig. 7a, some profiles of the vertical component, along the plate, at different distances from it are plotted. It is possible to point out how those structures smoothly merge together moving far from the porous plate, loosing the highest wavenumber mode, $e^{i \frac{2 \pi}{L} k x}$. The corresponding wavelength, $\lambda=L / k$ where $k$ is the wavenumber, instead, provides an estimation 


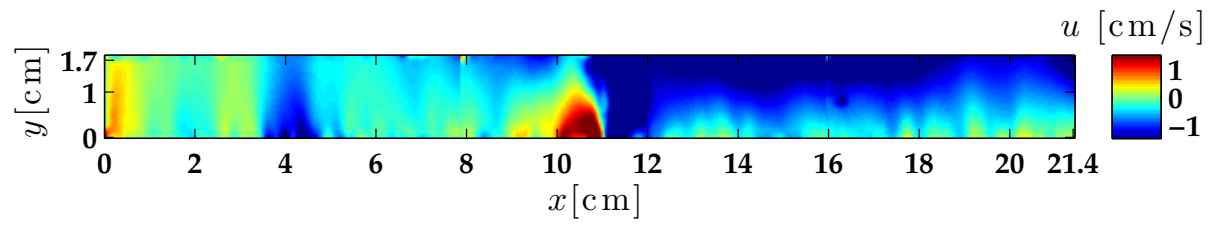

(a)

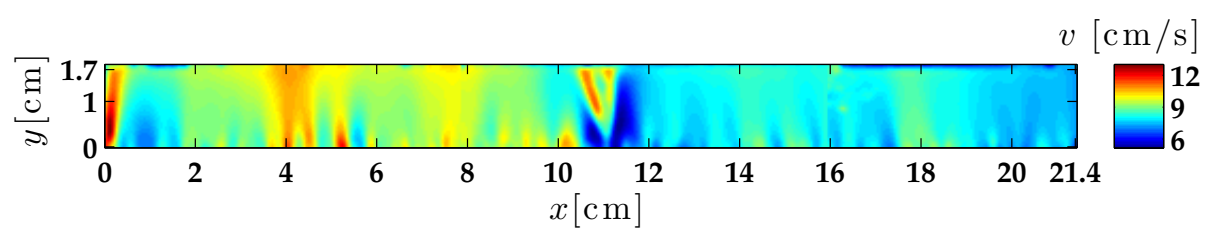

(b)

Fig. 5: Mean velocity field in the analysis of the inlet conditions at the exit of the porous plate, horizontal component (a) and vertical component (b)

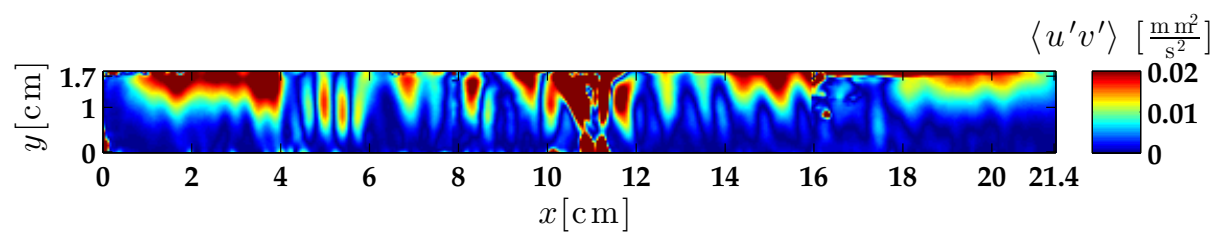

Fig. 6: Jet-like structures highlighted by the Reynolds stress $\left\langle u^{\prime} v^{\prime}\right\rangle$ in the inlet conditions analysis

of the initial size of those jet-like structures. Fig. 7b shows that these dimensions vary between $5 \mathrm{~mm}$ and $3 \mathrm{~cm}$.

\subsection{Closed-open configuration}

In this section, a comparison between the solutions of the models described in Sect. 2 and experimental data is reported. In order to do that, experimental data must be made dimensionless, so that the experimental injection velocity $U_{\text {inj }}$ is required. Since the experimental vertical velocity $v$ is not perfectly uniform (see Fig. $8 \mathrm{~b}$ ) as in the mathematical model, we derive the experimental $U_{\text {inj }}$ by the mass conservation law between the inlet from the plate, $\dot{m}=U_{\mathrm{inj}} L$, and a vertical line at the outlet chamber section, $\dot{m}=\int_{-H}^{0} u d y$, that reads

$$
U_{\mathrm{inj}}=\frac{1}{L} \int_{-H}^{0} u d y \approx 9.28 \mathrm{~cm} \mathrm{~s}^{-1}
$$

Actually, due to light reflections, $u$ is not available in a thin region near the upper and lower boundary, therefore in Eq. (18) the actual interval of integration is smaller than the whole height, but its contribute to the integral is negligible. 


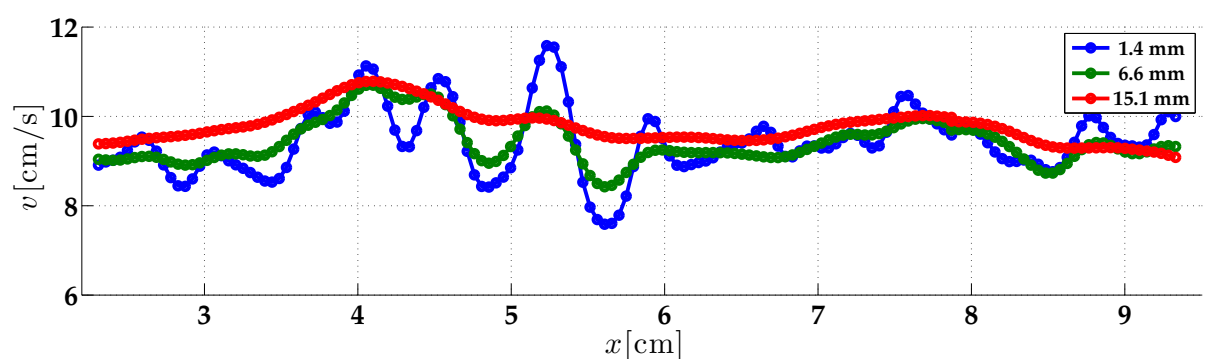

(a)

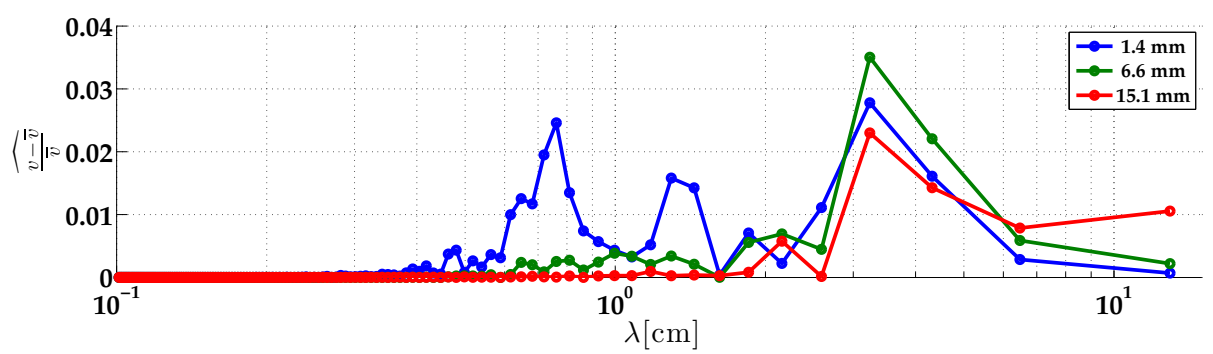

(b)

Fig. 7: Profiles of $v$ at several distances from the filter (a) and their Fourier transforms as functions of wavelength $\lambda(b)$

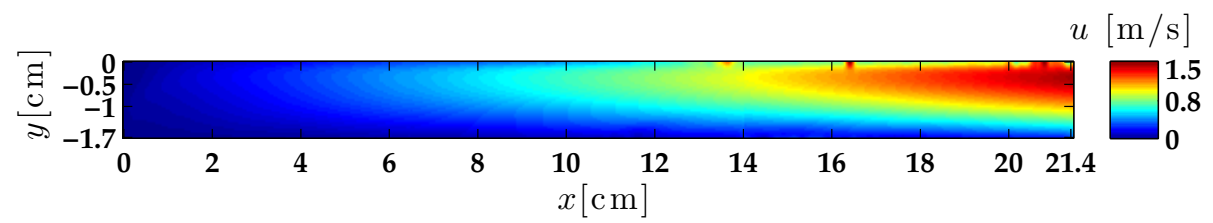

(a)

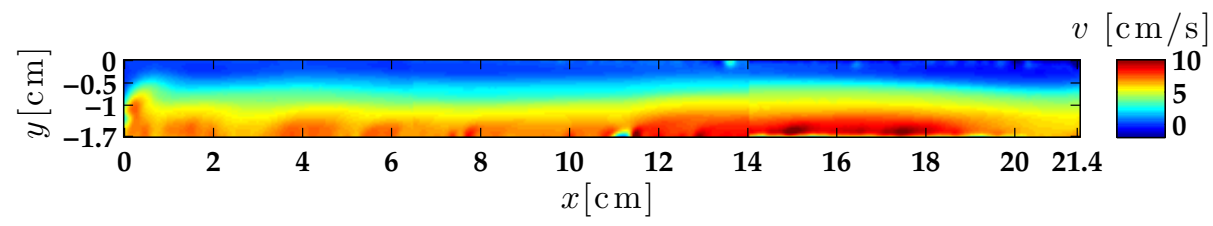

(b)

Fig. 8: Mean velocity field in the closed-open configuration

As reported from Eq. (18), the estimated value of $U_{\text {inj }}$ is $9.28 \mathrm{~cm} \mathrm{~s}^{-1}$, whereas the injection velocity derived from direct measurements at the inlet was $9.30 \mathrm{~cm} \mathrm{~s}^{-1}$. These values are very close, thus the system seems to be well controlled and the input condition is fully established. 


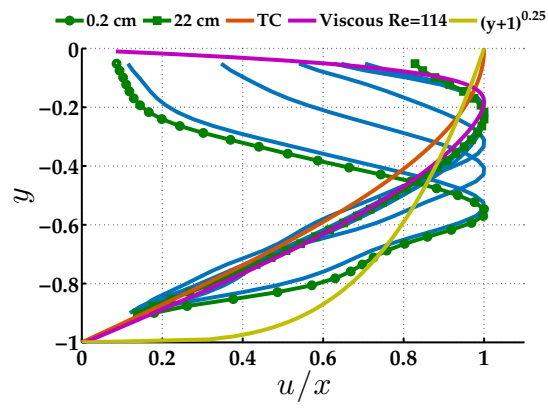

(a)

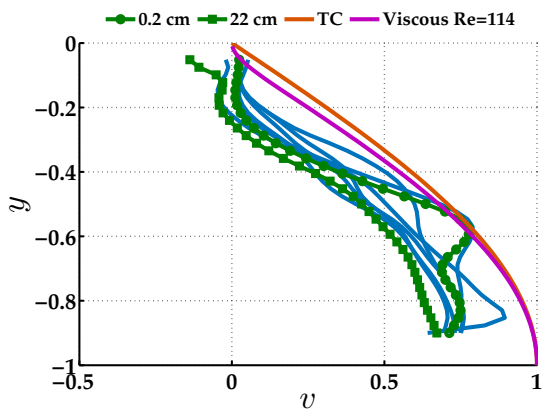

(b)

Fig. 9: Profiles of mean non-dimensional velocity at several positions along $x$-axis. Blue lines are the velocity profiles between the two green edges

Mean values The behavior of the two velocity components in the whole chamber is reported in Fig. 8a and 8b. In the first one, it is clear as the horizontal velocity increases when moving towards the right, as a consequence of the air inlet from the bottom and of the deviation due to the upper wall. Regarding the vertical velocity components, reported in Fig. 8b, little structures due to the porosity of the filter are still visible, even in this closed configuration. Note that the vertical velocity also increases when moving from left to right and that there is a lower layer in which the vertical component is predominant over the horizontal component and an upper layer where the opposite takes place. Note also that the order of magnitude is similar to the one obtained during the analysis of the inlet conditions in the previous section. We are not able to assess if the increase of the vertical velocity along the channel is related to the pressure drop, which is due to the development of $u$ and hence to the different pressure gradients across the filter experienced by the flow, or it is a consequence of the hydrodynamic instability as described in Chedevergne et al (2006).

Once data are made dimensionless ${ }^{2}$ with respect to $U_{\mathrm{inj}}$, we can compare them with (12), (15) and the numerical solution of (17). In Fig. 9 we reported some profile of velocity at several $x$ positions, in particular in Fig. 9a we plotted $u / x$ because analytical models are linear in $x$, while, in Fig. $9 \mathrm{~b}, v$ is independent of $x$. The solution of (17) has been obtained with $\mathrm{Re}_{\mathrm{inj}}=114$. From Fig. $9 \mathrm{a}$, it is clearly visible how the measured horizontal velocity profiles approach the viscous numerical solution much better than the inviscid ones. Indeed, as the Reynolds number is low, the no-slip boundary condition at the upper wall is not negligible and a thick boundary layer is obtained. This means that in such kind of flow configurations, the effect of viscosity cannot be neglected due to the presence of the upper wall. On the other hand, the vertical velocity component is less dependent on viscous effects being the highest values confined to the lower porous plate. In dimensionless variables, there is only a minor increase of the vertical velocity when moving towards the outlet cylinder section. In Fig. 9a, it is also possible to notice that near the closed head end of the cylinder, the horizontal profile is almost

\footnotetext{
2 Also the error should be scaled by $U_{\text {inj }}$. Then we obtain a maximum error, the one relative to the third section, of the order of 0.06 .
} 


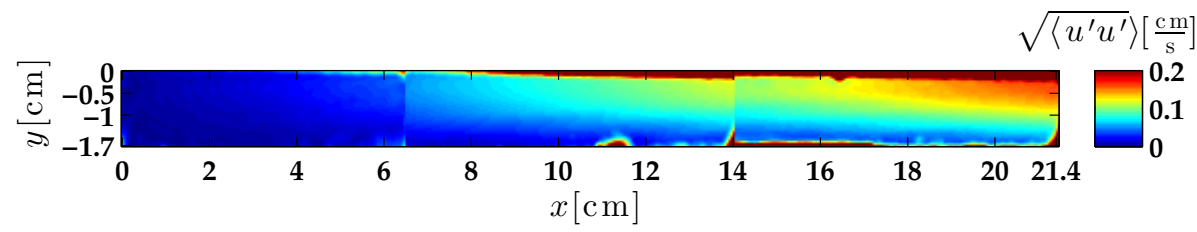

(a)

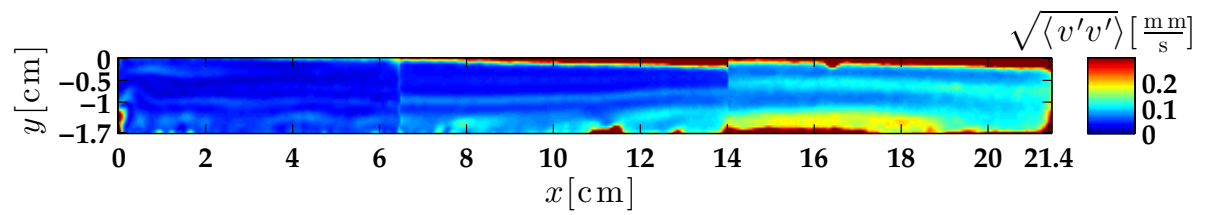

(b)

Fig. 10: Standard deviations of $u^{\prime},(\mathrm{a})$, and $v^{\prime},(\mathrm{b})$

symmetric, evolving in around a centimeter towards the non-symmetric behavior, which also results from analytical and numerical solutions. As in Yagodkin (1980)'s measurements, the present mean velocity profile does not seems to experience any transition to the turbulent shape, observed instead by Olson and Eckert (1966) and Huesmann and Eckert (1968) at the present Reynolds number. A possible explanation can reside in the level of pseudo-turbulence injected from the porous wall and the relative length of the channel, see also Beddini (1986). The pseudoturbulence is related to the level of spatial fluctuations introduced by the injection and can be estimated as follows

$$
\mathrm{PTI}=\sqrt{\frac{\frac{1}{N} \sum_{j=1}^{N}\left(v_{j}^{\mathrm{s}}-\overline{v^{\mathrm{s}}}\right)^{2}}{\bar{v}^{\mathrm{s}}}},
$$

where the superscript $\mathrm{s}$ indicates the velocity at the porous surface and $N$ is the number of data along the surface. The level of PTI in the present experiment is around 0.003 and, according to Beddini's results, it is too small to activate the transition to turbulence at the present Reynolds number.

Fluctuations The two standard deviations $\sqrt{\left\langle u^{\prime} u^{\prime}\right\rangle}$ and $\sqrt{\left\langle v^{\prime} v^{\prime}\right\rangle}$, which are the square root of the normal Reynolds stresses, are a statistical indication of how much each component of velocity fluctuates with respect to its mean value. Here, we obtained fluctuations of the order of millimeters per second for the velocity component $u$ and tenths of a millimeter per second for the $v$, as reported in Fig. 10. The amplitude of fluctuations is also increasing when moving towards the right part of the field, i.e. the outlet section. This means that the mean flow configuration reported in Fig. 8 is fluctuating or vertically (flapping) or horizontally (pressure waves) or both. It is important to understand if these fluctuations are related to vortices generated close to the plate and then convected towards the outlet. To this end, the detection of the mean vorticity field and of instantaneous flow structures is performed. In the mean vorticity field, reported in Fig. 11b, and furthermore in 


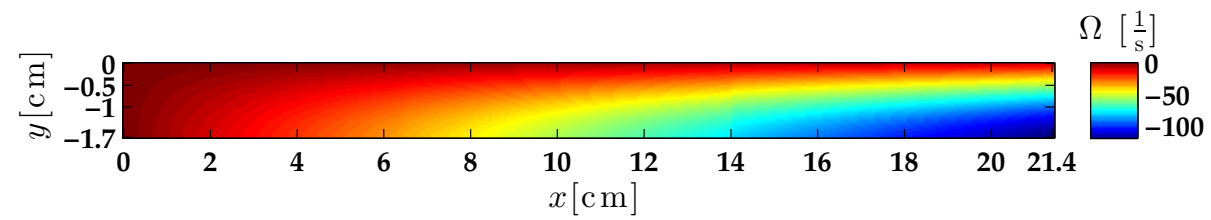

(a)

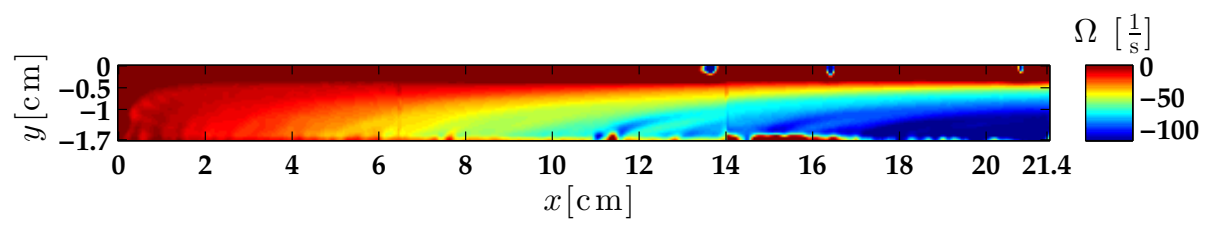

(b)

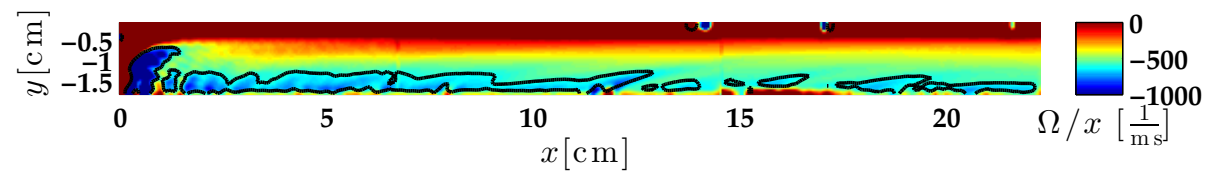

(c)

Fig. 11: Vorticity field of: TC solution (Eq. 16) (a), experimental data (b). Being $\Omega$ linear with $x$, spatial oscillations are highlighted by the $\Omega / x$ field in (c). The black solid line is an isoline of $\Omega / x=-610$

the $\Omega / x$ field reported in Fig. 11c, a spatially oscillating pattern, in the form of an array of crossflow jets, due to the jet-like structures generated by the porous plate is clearly visible. These structures are bigger than the single jets observed earlier, as they merge together when they are bended by the horizontal flow. They also become narrower as we go far from the head end of the rectangular cylinder.

It is plausible that the relatively high amplitude oscillations of $u$ are related more to these large scale structures rather than to the small vortical ones reported in Fig. 12. These small structures have been detected with the $\Delta$-criterion

$$
\Delta=P^{2}-4 Q<0
$$

where

$$
P=\frac{\partial u}{\partial x}+\frac{\partial v}{\partial y} \quad \text { and } \quad Q=\frac{\partial u}{\partial x} \frac{\partial v}{\partial y}-\frac{\partial u}{\partial y} \frac{\partial v}{\partial x}
$$

as prescribed by Jeong and Hussain (1995). Looking at Fig. 12, it is clear that vortical structures are mainly generated at the lower porous plate, presumably due to the jet-like structures interactions. These vortices grow in number, size and intensity when moving towards the right outlet part and contribute to the increasing of fluctuations levels mostly on the outlet part of the chamber.

In order to establish whether flow transition to turbulence appears, Yamada and Ishikawa (1976) compared turbulence intensity peak, i.e. $I_{\mathrm{p}}=\max _{y} I=$ $\max _{y} \sqrt{\left\langle u^{\prime} u^{\prime}\right\rangle+\left\langle v^{\prime} v^{\prime}\right\rangle}$, with an horizontal velocity reference, e.g. $\max _{y} u$. Since 


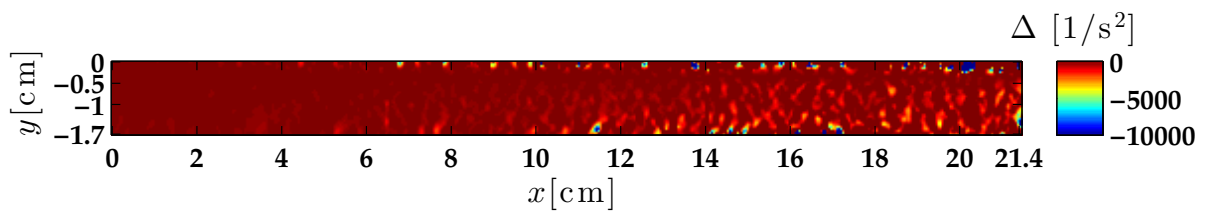

Fig. 12: Instantaneous vortical structures highlighted with values of $\Delta<0$. Dark red is greater than zero, while lighter colors are negative values

they adopted injection velocities higher than $0.5 \mathrm{~m} \mathrm{~s}^{-1}$, their results are not comparable with the one presented herein and no significant intensity peak has been found. However, the turbulence intensity of the present data is almost equal to the rms of $u^{\prime}, \sqrt{\left\langle u^{\prime} u^{\prime}\right\rangle}$. Indeed, as it will be shown below with the analysis of $u^{\prime}$ and $v^{\prime}$, the main contribution to fluctuation is the linear increase of horizontal velocity that, as a consequence, enlarges its fluctuations too.

Given a time series $\left\{u_{n}^{\prime}\right\}_{n=1}^{N_{\mathrm{im}}}$, where $n$ denotes the $n$-th instantaneous field of $N_{\text {im }}$, its Probability Density Function (PDF) is defined as

$$
\operatorname{PDF}\left(u^{\prime}\right)_{i}=\frac{\operatorname{hist}\left(u^{\prime}\right)_{i}}{\frac{\max _{n} u^{\prime}-\min _{n} u^{\prime}}{N_{\text {bin }}} \sum_{j=1}^{N_{\text {bin }}} \operatorname{hist}\left(u^{\prime}\right)_{j}}, \quad \text { for } j=1, \ldots, N_{\text {bin }},
$$

where $\operatorname{hist}\left(u^{\prime}\right)_{j}=\#\left\{u_{i}^{\prime} \in B_{j}\right.$ for $\left.i=1, \ldots, N_{\mathrm{im}}\right\}$ is the number of velocity data that lies into the $j$-th bin and $N_{\text {bin }}$ is the total number of bins. The PDF of both velocity components have been computed at different locations of the rectangular cylinder. For the horizontal component, $u^{\prime}$, the PDFs in the upper region of the chamber are barely comparable with a normal distribution, unlike in the region closer to the porous plate, as shown in Fig. 13. The vertical component, $v^{\prime}$, instead, follows the Gaussian law everywhere

$$
\mathcal{N}(\mu, \sigma) \sim \frac{1}{\sigma \sqrt{2 \pi}} e^{-\frac{1}{2}\left(\frac{x-\mu}{\sigma}\right)^{2}}
$$

as reported in Fig. 14. This indicates that, where the velocity magnitude is small, fluctuations look like white noise on both velocity components. The vertical component fluctuations are everywhere of the same order of magnitude of the error $\left(\approx 5 \mathrm{~mm} \mathrm{~s}^{-1}\right)$, therefore it is plausible that the observed gaussian distribution is the distribution of the error. On the other hand, the bimodal behavior of the $u$ component in the upper region shows, with longitudinal oscillations of one order of magnitude greater than transversal ones, that incoherent (Gaussian) fluctuations feed longitudinal, coherent fluctuations. Moreover, the spatial distributions of $u^{\prime}$ and $v^{\prime}$ have been reported in Fig. 15 and compared respectively with the first acoustic horizontal eigenmode, $h_{1}=A_{0} \sin \left(\frac{2 \pi}{4 L} x\right)$ and the fourth vertical eigenmode, $v_{4}=A_{0} \sin \left(4 \frac{2 \pi}{2 L} x\right)$, where $A_{0}$ is an amplitude comparable with the experimental data. The horizontal perturbation, even though it fluctuates periodically as stated by the PDF, has a linear profile along $x$ instead of sinusoidal. Despite the vertical perturbations are of the order of the error, its profile shows the oscillatory behavior as a combination of the first four modes, even if it is not evident with how many nodes. 


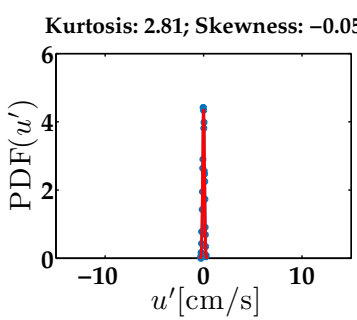

(a)

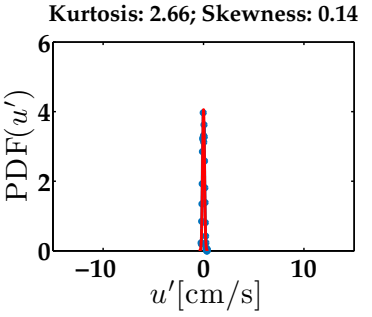

(d)

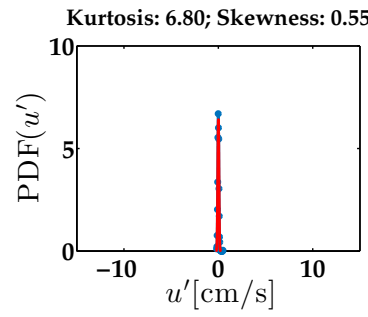

(g)

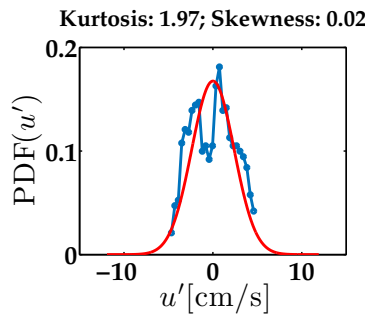

(b)

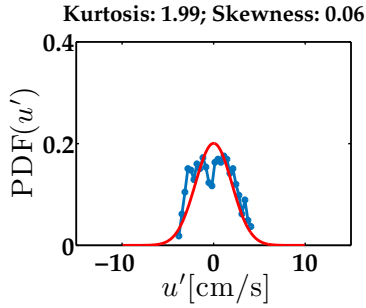

(e)

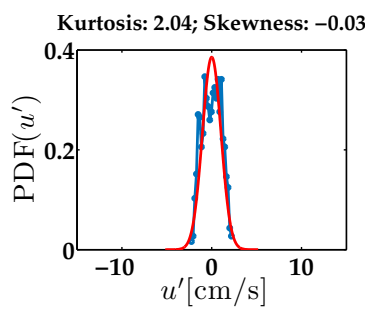

(h)

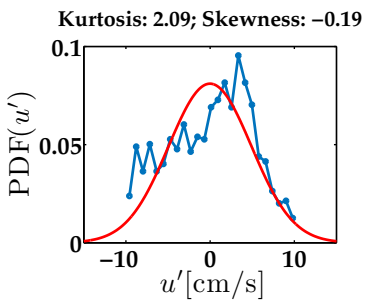

(c)

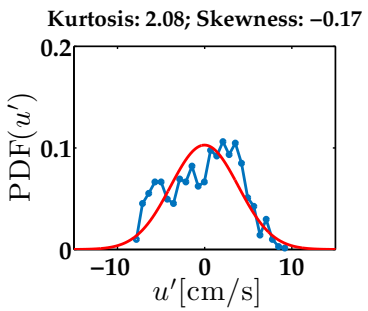

(f)

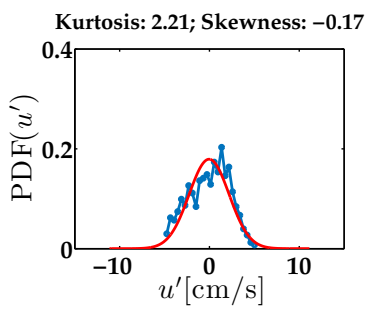

(i)

Fig. 13: Probability density function of $u^{\prime}$ at the nine positions indicated by letters (a, b, c etc.) in Fig. 3. In particular, subfigure (a) corresponds to data at the point labels with (a) and so on

Transversal planes In order to assess how much three-dimensionality can affect PIV measurements on the two-dimensional plane, we analyzed three transversal planes $(z, y)$ at $x=41 \mathrm{~mm}, x=90 \mathrm{~mm}$ and $x=144 \mathrm{~mm}$. Since the orthogonal velocity, $u$, is linear with the $x$, it had been impossible to measure planes for higher values of $x$, because particles escaped from the light sheet too fast.

The vertical component of velocity, $v$, except for the left and right end, has the same behavior obtained in the $(x, y)$ plane, i.e. it shows jet-like structures, see Fig. 16a-16e. Note that because of particles easily escaping from the measuring plane, we had been forced to reduce the delay between two consecutive images, consequently reducing the maximum displacement of each particle. This enlarges the uncertainty on the experimental data, which is inversely proportional to the $\Delta t$ used. Fig. 16b-16f show that the flow does not cross the main plane $(x, y)$ 


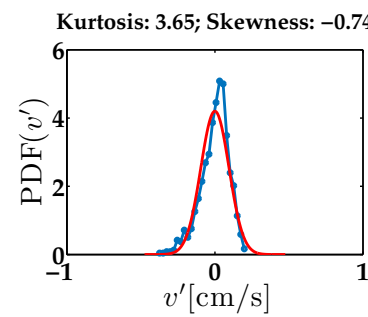

(a)

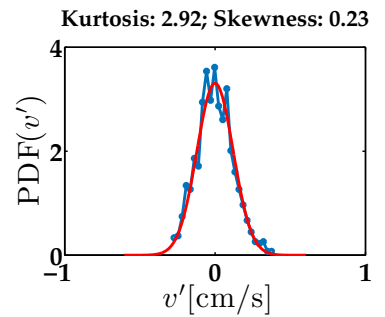

(d)

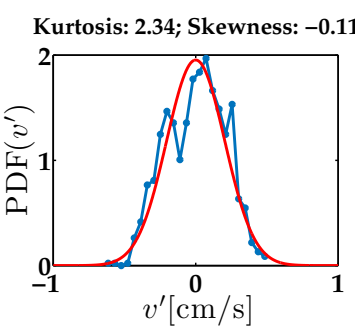

(g)

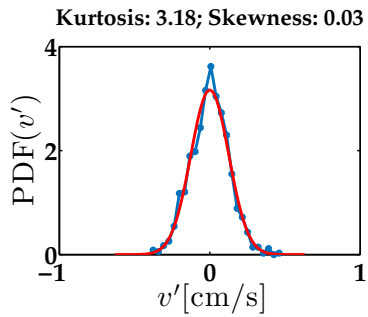

(b)

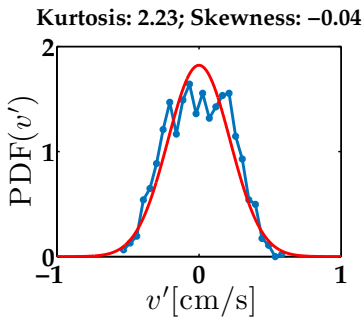

(e)

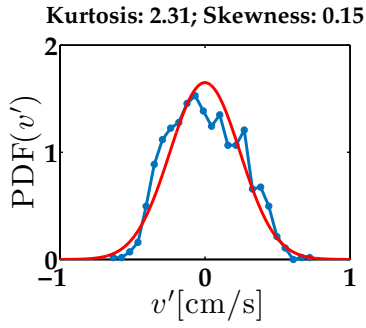

(h)

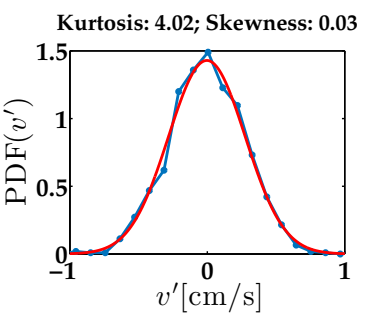

(c)

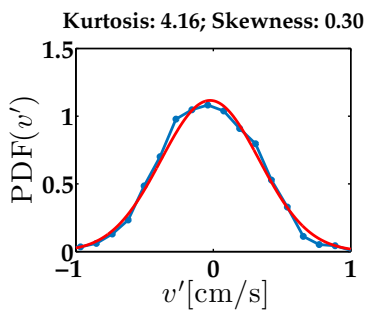

(f)

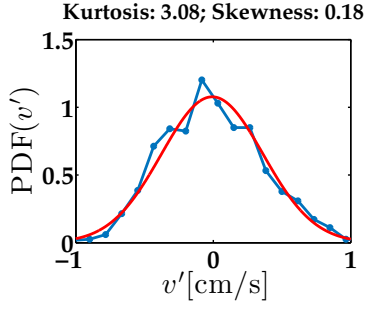

(i)

Fig. 14: Probability density function of $v^{\prime}$ at the nine positions indicated by letters (a, b, c etc.) in Fig. 3. In particular, subfigure (a) corresponds to data at the point labels with (a) and so on

at $z=0$, but the contribution of $w$, which is due somehow to the two lateral boundaries, becomes larger moving downstream.

\section{Remarks and conclusions}

In this paper, a characterization of the flow inside a rectangular cylinder, in which the flow enters from a bottom porous plate and exits from one side, has been presented. The velocity field was obtained by means of Particle Image Velocimetry, in order to get instantaneous and averaged flow field spatial configurations. Samples made up of 1000 image couples were used to compute statistical moments. Preliminarily, the flow generated by pressure difference between two side of a porous material with porosity of $20 \mu \mathrm{m}$ has been characterized. A pattern of 


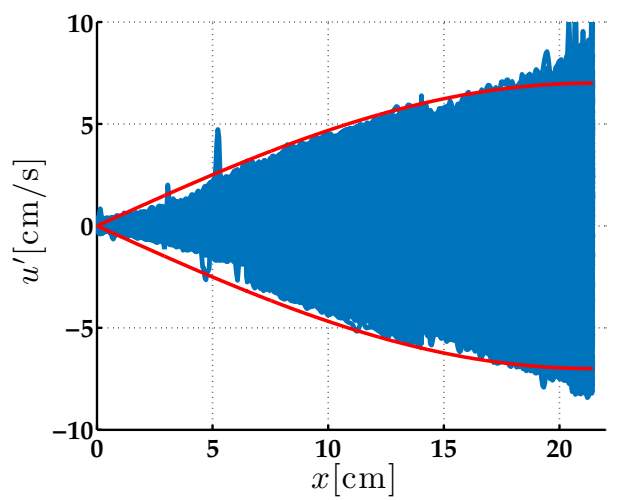

(a)

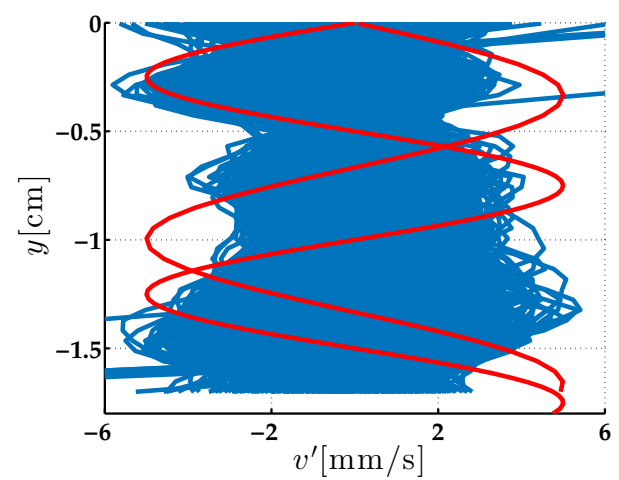

(b)

Fig. 15: Superposition of the instantaneous $u^{\prime}(x)$ at $y=-0.9 \mathrm{~cm}$ (a) and of $v^{\prime}(y)$ at $x=0.4 \mathrm{~cm} \mathrm{(b)}$

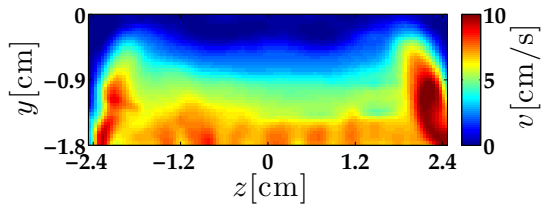

(a)

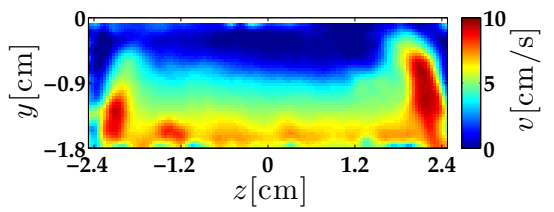

(c)

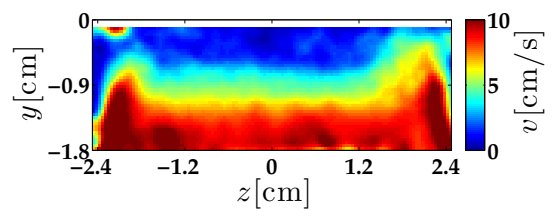

(e)

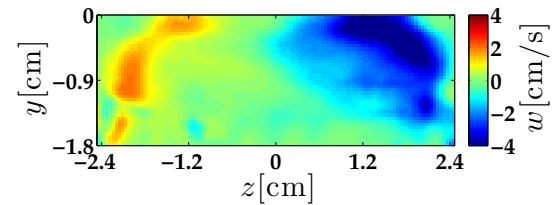

(b)

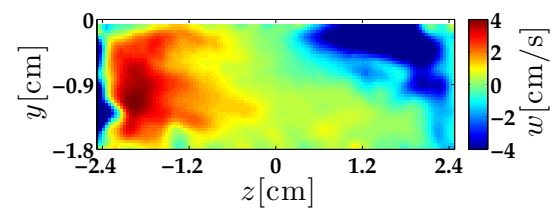

(d)

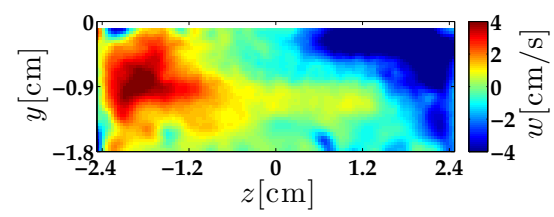

(f)

Fig. 16: Velocity $v$ (first column) and $w$ (second column) on transversal planes at several positions along $x$ : (a) and (b) at $4.1 \mathrm{~cm},(\mathrm{c})$ and (d) at $9.0 \mathrm{~cm}$ and (e) and (f) at $14.4 \mathrm{~cm}$

jet-like structures with dimensions of roughly $5 \mathrm{~mm}$ at a distance from the filter of approximately $2 \mathrm{~mm}$ has been found, which must be considered as the inlet condition in the test section. The same structures were found in the closed config- 
uration, but are only visible in the $y$-component of the velocity, which is normal to the porous plate and must be considered as the primary flow.

Mean values, standard deviations and probability density functions of both $u$ and $v$ have been computed. The increment of velocity magnitude, especially due to the horizontal component, as also the increasing values of fluctuations, when moving towards the outlet section, suggests that a relevant contribution to unsteadiness is due to turbulence generated by parallel jets and boundary layer, respectively on the lower and upper surfaces. Moreover, we have shown a snapshot of vortical structures captured in one of the instantaneous field, pointing out how these structures are growing in number, size and intensity when moving towards the outlet. These are the spatial traces of the developing turbulence on the lower and upper surfaces. Anyhow, according to the experiments of Yagodkin (1980) but in contrast with Olson and Eckert (1966) and Huesmann and Eckert (1968), no transition of the mean velocity profile has been found. This could be explained by taking into account the levels of pseudo-turbulence intensity of the injected flow as explained by Beddini (1986).

We compared the mean values obtained from the experimental data with analytical models. Since the injection Reynolds number is low, $R e \approx 110$, the most accurate model is the one that takes into account the boundary layer at the top wall. In any case, all models are not able to predict the amount of fluctuations or the parallel jet flow configuration derived from the measurements. As a result of the analysis of the probability density functions of $u^{\prime}$ and $v^{\prime}$, we found two kind of oscillations, one on the $u$ near the exit and another, similar to Gaussian noise, elsewhere. We do not believe that this Gaussian noise is due the to the sprouting of the little vortices observed, because it is present also beforehand the onset of these vortices. It is more plausible that it is due to the error, which is in addition of the same order of magnitude.

We must observe that the primary obstacle of this kind of measurements is the extremely high difference between the order of magnitude of the vertical and horizontal component. In any case, the developing vortices contribute to the enhanced level of fluctuations must be considered in proper modeling of this type of flow. In particular, coherent horizontal fluctuations should be obtained.

\section{References}

Avalon G, Casalis G, Griffond J (1998) Flow instabilities and acoustic resonance of channels with wall injection, American Institute of Aeronautics and Astronautics. DOI doi:10.2514/6.1998-3218, URL http://dx.doi.org/10.2514/6.1998-3218

Avalon G, Ugurtas B, Grisch F, Bresson A (2001) Numerical computations and visualization tests of the flow inside a cold gas simulation with characterization of a parietal vortex shedding. Tiré à part - Office national d'études et de recherches aerospatiales

Beddini RA (1986) Injection-induced flows in porous-walled ducts. AIAA Journal 24(11):17661773, DOI 10.2514/3.9522, URL http://dx.doi.org/10.2514/3.9522

Casalis G, Avalon G, Pineau JP (1998) Spatial instability of planar channel flow with fluid injection through porous walls. Physics of Fluids (1994-present) 10(10):2558-2568

Casalis G, Chedevergne F, Feraille T, Avalon G (2006) A new stability approach for the flow induced by wall injection. Springer

Chedevergne F, Casalis G, Feraille T (2006) Biglobal linear stability analysis of the flow induced by wall injection. Physics of Fluids (1994-present) 18(1):14-103

Culick F (1966) Rotational axisymmetric mean flow and damping of acoustic waves in asolid propellant rocket. AIAA Journal 4(8):1462-1464 
Féraille T, Casalis G (2005) Global stability of the flow induced by wall injection. Tiré à part - Office national d'études et de recherches aerospatiales

Flandro GA (1986) Vortex driving mechanism in oscillatory rocket flows. Journal of Propulsion and Power 2(3):206-214, DOI 10.2514/3.22871, URL https://doi.org/10.2514/3.22871

Griffond J, Casalis G, Pineau JP (2000) Spatial instability of flow in a semiinfinite cylinder with fluid injection through its porous walls. European Journal of Mechanics B/Fluids 19(1):69 - 87, DOI http://dx.doi.org/10.1016/S0997-7546(00)00105-9, URL http://www.sciencedirect.com/science/article/pii/S0997754600001059

Hart RW, McClure FT (1959) Combustion instability: Acoustic interaction with a burning propellant surface. The Journal of Chemical Physics 30(6):1501-1514, DOI http://dx.doi.org/10.1063/1.1730226, URL http://scitation.aip.org/content/aip/journal/jcp/30/6/10.1063/1.1730226

Huesmann K, Eckert ERG (1968) Studies of the laminar flow and the transition to turbulence in porous tubes with uniform injection through the tube wall. Warme-und Stoffubertragung

Jeong J, Hussain F (1995) On the identification of a vortex. Journal of Fluid Mechanics 285:69-94, DOI 10.1017/S0022112095000462, URL https://www.cambridge.org/core/article/div-class-title-on-the-identification-of-a-vortexdiv/D26006DDB95FB28DA80E28A581182DF1

Laboureur D, Tóth B, Anthoine J (2010) Investigation of the taylor-culick flow through particle image velocimetry and numerical simulation. AIAA Journal 48(6):1077-1084, DOI 10.2514/1.44616, URL http://dx.doi.org/10.2514/1.44616

Majdalani J, Fist A (2014) Improved mean flow solution for solid rocket motors with a naturally developing swirling motion. In: Propulsion and Energy Forum, AIAA

McClure FT, Lure FT, Cantrell RH, Hart RW (1963) Interaction between sound and flow: Stability of T-burners. AIAA Journal 1(3):586-590, DOI 10.2514/3.54846, URL http://dx.doi.org/10.2514/3.54846

Olson RM, Eckert ERG (1966) Experimental studies of turbulent flow in a porous circular tube with uniform fluid injection through the tube wall. Journal of Applied Mechanics 33(1):7-17, URL http://dx.doi.org/10.1115/1.3625030

Perrotta A, Gnisci S, Romano GP (2017) Resolving the flow fine structure generated downstream a permeable plate using single-pixel correlation in comparison to window correlation. In: The 12th International Symposium on Particle Image Velocimetry

Pimenta M, Moffat RJ (1974) Stability of flow through porous plates: Coalescent jets effect. AIAA Journal 12(10):1438-1440, DOI 10.2514/3.49512, URL http://dx.doi.org/10.2514/3.49512

Taylor G (1956) Fluid flow in regions bounded by porous surfaces. Proceedings of the Royal Society of London Series A Mathematical and Physical Sciences 234(1199):456-475

Thielicke W, Stamhuis E (2014) Pivlab-towards user-friendly, affordable and accurate digital particle image velocimetry in matlab. Journal of Open Research Software 2(1):p.e30, DOI http://doi.org/10.5334/jors.bl

Varapaev V, Yagodkin V (1969) Flow stability in a channel with porous walls. Fluid Dynamics 4(5):60-62

Yagodkin V (1980) Use of channels with porous walls for studying flows which occur during combustion of solid propellants. Tech. rep.

Yamada K, Ishikawa N (1976) Simulative study on the erosive burning of solid rocket motors. AIAA Journal 14(9):1170-1176, DOI 10.2514/3.61451, URL https://arc.aiaa.org/doi/abs/10.2514/3.61451 